\title{
The International Standards Booklet for Neurological and Functional Classification of Spinal Cord Injury*
}

\author{
J F Ditunno Jr MD,${ }^{1}$ W Young PhD MD,${ }^{2}$ W H Donovan MD,${ }^{3}$ G Creasey MD ${ }^{4}$ \\ 'Department of Rehabilitation Medicine, Thomas Jefferson University Hospital, \\ Philadelphia, Pennsylvania; ${ }^{2}$ Department of Neurosurgery, New York University Medical \\ Center, New York; ${ }^{3}$ The Institute for Rehabilitation and Research, Houston, Texas; \\ ${ }^{4}$ Department of Biomedical Engineering, Case Western Reserve University, Cleveland, \\ Ohio, USA.
}

\section{Introduction}

For almost a quarter of a century the international community in spinal cord injury (SCI) has advocated a uniform measure of SCI severity. ${ }^{1}$ Such agreement is needed for the accurate communication between clinicians and for comparisons of research results among investigators. The severity of the injury after SCI is primarily reflected in the extent of paralysis and loss of sensation (impairment) and the inability to perform activities of daily living (disability). These measurements of impairment and disability serve as the determinants of the clinical outcome in SCI. ${ }^{2}$ The importance of clinical outcome measures have been emphasized for purposes of cost justification in the United States, in recent international neurotrauma research forums, and particularly in multicenter clinical trials. A minimum data set which is both practical and reliable is essential to multicenter trials. The use of such measures in recent studies includes the prognosis of motor recovery in the upper extremities of tetraplegic subjects based on an increase of muscle strength and motor levels ${ }^{3}$ the effects of drug intervention in SCI based on improvement in motor and sensory scores, ${ }^{4,5}$ and the efficiency of rehabilitation in SCI based on improvement of functional assessment measures. ${ }^{6}$ In response to this need, the American Spinal Injury Association (ASIA) initially developed standards for neurological classification of spinal cord injury in $1982 .^{7}$ In

*This is published with the permission of the American Spinal Injury Association (ASIA). recognition of the need for greater acceptance, ASIA sought input from a broader array of experts representing SCI research interests, as well as from diverse clinical specialists. The international Medical Society of Paraplegia (IMSOP), as well as many other organizations and professional societies interested in classification, were represented by members on the committee formed in 1990. The international standards revised by the ASIA Committee in 1992 were endorsed by IMSOP in Barcelona, in September 1992.

The rationale for these standards evolved from two major influences. The first was the need to refine the definition of neurological levels. For close to 30 years rehabilitation and SCI clinicians used the neurological level to define function or disability. ${ }^{8}$ Key muscles and key sensory points were developed to determine these levels more precisely for the national database of the Model SCI Centers' reporting purposes. ${ }^{9}$ The second influence was the need for agreement between investigators on key muscles and key sensory points for use as endpoints in motor and sensory scores. Several of the authors recognized this need ${ }^{10}$ and the opportunity to achieve it for use in clinical trials. The need for uniformity was particularly evident when it became apparent that two of the largest study groups involved in acute spinal cord injury, the National Database of the Model Spinal Cord Injury Centers, and the National Acute Spinal Cord Injury Study, ${ }^{11}$ were using somewhat disparate methods for collecting neurological data. Because the key muscles and their five grades are used to determine both 
neurological levels and to calculate the motor score, lengthy discussions were required by the ASIA Committee to achieve agreement. Each of the measures in the standards, however, has gone through a laborious process of discussion and documentation during meetings, conference calls, input from national and international societies and organizations, and we shall continue to invite comments and recommendations. Thus, the face validity of the standards has been arrived at by this process. Dr Wise Young assisted the primary author as co-chairman, and Dr William Donovan served as co-editor. Drs Paul Dollfus and Hans Frankel identified Dr Graham Creasey to provide input from IMSOP. The remainder of the committee members include Drs Michael B Bracken, Margaret Brown, Thomas B Ducker, Frederick M Maynard Jr, Samuel L Stover, Charles H Tator, Robert L Waters, and Jack E Wilberger. Further refinements of validity and precision will be accomplished by an annual review of research findings, comments and the booklet will be updated as necessary. In an effort to increase the accuracy and the reliability of the examination, a teaching package, which will provide a manual and video tapes demonstrating the examination, scoring, scaling and rationale of the measures, is in preparation. The initial draft video of the examination has been shown in North America, Asia, and twice in Europe this spring and summer, and has been greeted with enthusiasm. It should be available in 1994. These standards represent the most valid, precise, and reliable minimum data set and currently are being utilized by the National Spinal Cord Injury database and two large multicenter drug trials involving more than forty SCI centers in the United States and Canada. IMSOP is committed to promulgating these standards for international use and is responsible for approving all translations of this booklet. ${ }^{12}$ These standards are published with the permission of the American Spinal Injury Association and copies of the booklet and training package can be obtained by contacting: Lesley $M$ Hudson MA, 2020 Peachtree Road, NW Atlanta, Georgia 30309, USA.

\section{Definitions}

Tetraplegia (preferred to 'quadriplegia')

This term refers to impairment or loss of motor and/or sensory function in the cervical segments of the spinal cord due to damage of neural elements within the spinal canal. Tetraplegia results in impairment of function in the arms as well as in the trunk, legs and pelvic organs. It does not include brachial plexus lesions or injury to peripheral nerves outside the neural canal.

\section{Paraplegia}

This term refers to impairment or loss of motor and/or sensory function in the thoracic, lumbar or sacral (but not cervical) segments of the spinal cord, secondary to damage of neural elements within the spinal canal. With paraplegia, arm functioning is spared, but, depending on the level of injury, the trunk, legs and pelvic organs may be involved. The term is used in referring to cauda equina and conus medullaris injuries, but not to lumbosacral plexus lesions or injury to peripheral nerves outside the neural canal.

\section{Quadriparesis and paraparesis}

Use of these terms is discouraged, as they describe incomplete lesions imprecisely. Instead, the ASIA Impairment Scale (see below) provides a more precise approach.

\section{Dermatome}

This term refers to the area of the skin innervated by the sensory axons within each segmental nerve (root).

\section{Myotome}

This term refers to the collection of muscle fibers innervated by the motor axons within each segmental nerve (root).

Neurological level, sensory level and motor level (see summary chart)

The first of these terms refers to the most caudal segment of the spinal cord with normal sensory and motor function on both sides of the body. In fact, the segments at which normal function is found often differ 
by side of body and in terms of sensory vs motor testing. Thus, up to four different segments may be identified in determining the neurological level, i.e., R-sensory, Lsensory, R-motor, L-motor. In cases such as this, it is strongly recommended that each of these segments be separately recorded and that a single 'level' not be used, as this can be misleading in such cases. When the term 'sensory level' is used, it refers to the most caudal segment of the spinal cord with normal sensory function on both sides of the body; the motor level is similarly defined with respect to motor function. These 'levels' are determined by neurological examination of: (1) a key sensory point within each of 28 dermatomes on the right and 28 dermatomes on the left side of the body, and (2) a key muscle within each of 10 myotomes on the right and 10 myotomes on the left side of the body.

\section{Skeletal level}

This term refers to the level at which, by radiographic examination, the greatest vertebral damage is found.

Sensory scores and motor scores (see summary chart)

Numerical summary scores that reflect the degree of neurological impairment associated with the SCI.

\section{Incomplete injury}

If partial preservation of sensory and/or motor functions is found below the neurological level and includes the lowest sacral segment, the injury is defined as incomplete. Sacral sensation includes sensation at the anal mucocutaneous junction as well as deep anal sensation. The test of motor function is the presence of voluntary contraction of the external anal sphincter upon digital examination.

\section{Complete injury}

This term is used when there is an absence of sensory and motor function in the lowest sacral segment.

\section{Zone of partial preservation (ZPP)}

This term refers to those dermatomes and myotomes caudal to the neurological level that remain partially innervated. When some impaired sensory and/or motor function is found below the lowest normal segment, the exact number of segments so affected should be recorded for both sides as the ZPP. The term is used only with complete injuries.

\section{Neurological examination}

\section{Introduction}

The neurological examination has two components (sensory and motor), which are separately described below. Further, the neurological examination has both required as well as optional, though recommended, elements. The required elements are used in determining the sensory/motor/neurological levels, in generating scores to characterize sensory/motor functioning and in determining completeness of the injury. The optional measures, though not used in scoring, may add to a specific patient's clinical description.

\section{When the patient is not fully testable}

When a key sensory point or key muscle is not testable for any reason, the examiner should record ' $\mathrm{NT}$ ' instead of a numeric score. In such cases, sensory and motor scores for the affected side of the body, as well as total sensory and motor scores, cannot be generated with respect to the injury at that point in treatment. Further, when associated injuries, e.g., traumatic brain injury, brachial plexus injury, limb fracture, etc., interfere with completion of the neurological examination, the neurological level should still be determined as accurately as possible. However, obtaining the sensory/motor scores and impairment grades should be deferred to later examinations.

\section{Sensory examination: required elements}

The required portion of the sensory examination is completed through the testing of a key point in each of the 28 dermatomes on the right and on the left sides of the body. 
At each of these key points, two aspects of sensation are examined: sensitivity to pin prick and to light touch. Appreciation of pin prick and of light touch at each of the key points is separately scored on a three-point scale:

$$
\begin{aligned}
0= & \text { absent } \\
1= & \text { impaired } \\
& \quad \text { (partial or altered appreciation, } \\
& \text { including hyperaesthesia) } \\
2= & \text { normal } \\
\mathrm{NT}= & \text { not testable }
\end{aligned}
$$

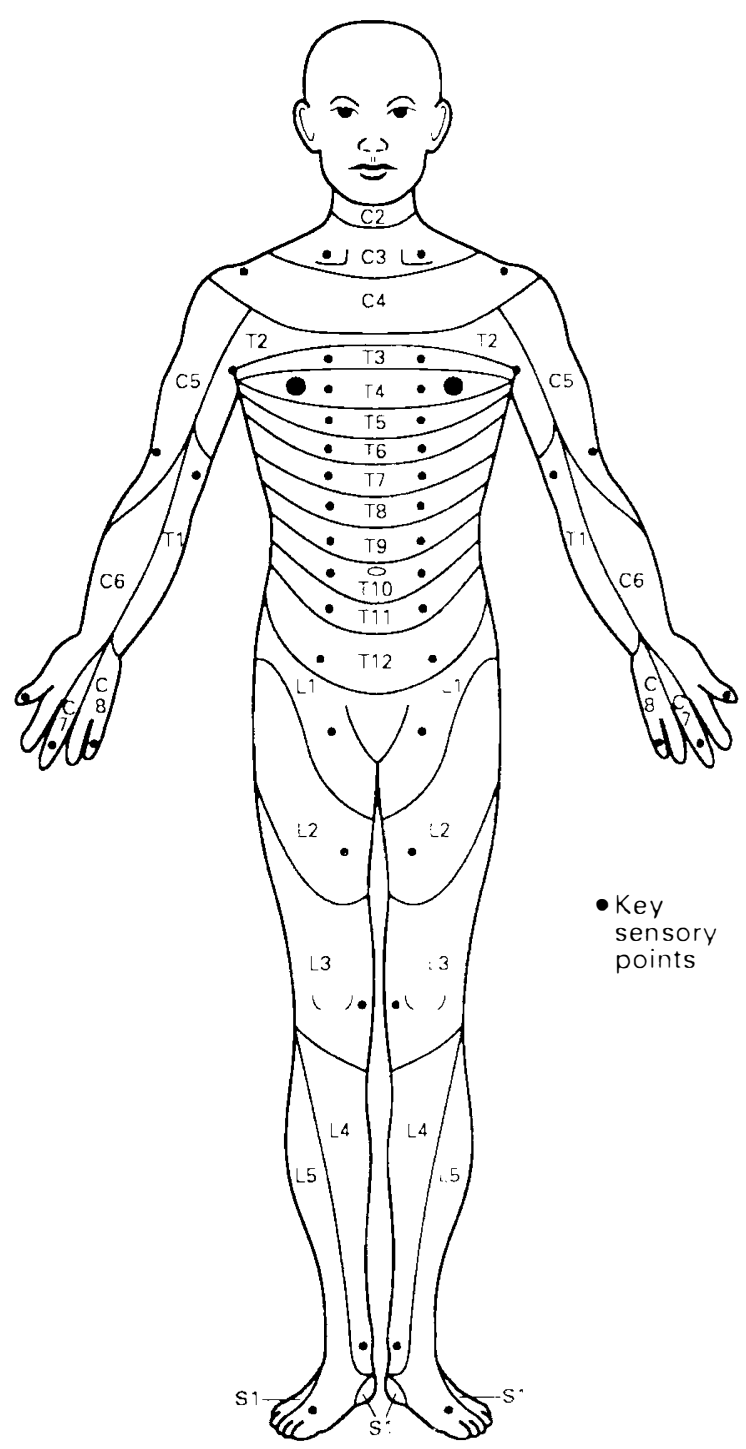

The testing for pin sensation is usually performed with a disposable safety pin; light touch is tested with cotton. In testing for pin appreciation, the inability to distinguish between dull and sharp sensation is graded as 0 .

The following key points are to be tested bilaterally for sensitivity (Fig 1 and diagram on summary chart). Asterisks (see below) indicate that the point is at the mid-clavicular line:

\section{C2-Occipital protuberance}
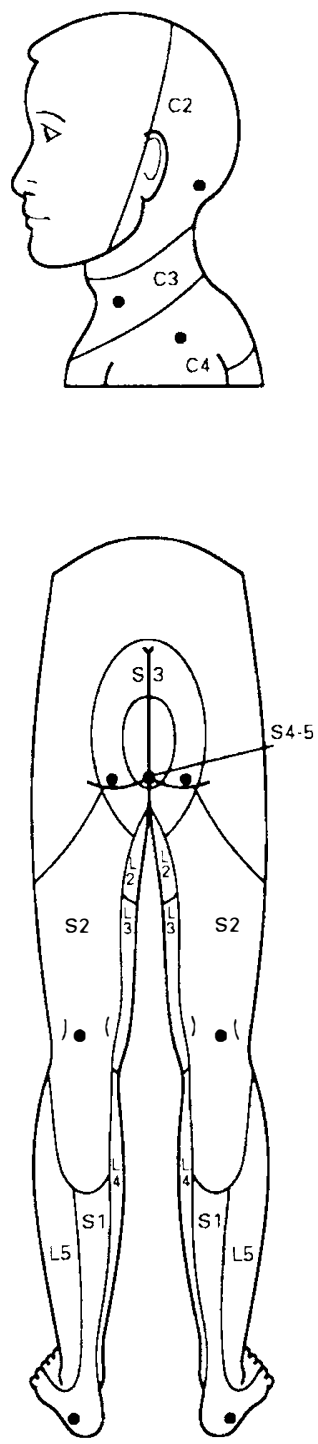

Figure 1 Key sensory points. 
C3-Supraclavicular fossa

C4-Top of the acromioclavicular joint

C5-Lateral side of the antecubital fossa

C6-Thumb

C7-Middle finger

C8-Little finger

T1-Medial (ulnar) side of the antecubital fossa

T2-Apex of the axilla

T3-Third intercostal space (IS)*

T4-Fourth IS (nipple line)*

T5-Fifth IS (midway between T4 and T6)*

T6-Sixth IS (level of xiphisternum)*

T7-Seventh IS (midway between T6 and T8)*

T8-Eighth IS (midway between T6 and T10)*

T9-Ninth IS (midway between T8 and $\mathrm{T} 10)^{*}$

T10-Tenth IS (umbilicus)*

T11-Eleventh IS (midway between T10 and $\mathrm{T} 12)^{*}$

T12-Inguinal ligament at mid-point

L1 - Half the distance between T12 and L2

L2-Mid-anterior thigh

L3-Medial femoral condyle

L4-Medial malleolus

L5-Dorsum of the foot at the third metatarsal phalangeal joint

S1 - Lateral heel

S2-Popliteal fossa in the mid-line

S3-Ischial tuberosity

S4-5-Perianal area (taken as one level)

In addition to bilateral testing of these key points, the external anal sphincter should be tested through insertion of the examiner's finger; perceived sensation should be graded as being present or absent (i.e., enter Yes or No on the patient's summary chart). This information is needed in determining completeness/incompleteness of injury.

\section{Sensory examination: optional elements}

For purposes of SCI evaluation, the following aspects of sensory function are defined as optional (though they are strongly recommended): position sense and awareness of deep pressure/deep pain. If these are examined, it is recommended that they be graded using the sensory scale below, (ab- sent, impaired, normal). It is also suggested that only one joint be tested for each extremity; the index finger and the great toe of the right and left sides are recommended.

\section{Motor examination: required elements}

The required portion of the motor examination is completed through the testing of a key muscle (one on the right and one on the left side of the body) in the 10 paired myotomes (see below). Each key muscle should be examined in a rostral-caudal sequence.

The strength of each muscle is graded on a six-point scale.

$0=$ total paralysis

$1=$ palpable or visible contraction

$2=$ active movement, full range of motion (ROM) with gravity eliminated

$3=$ active movement, full ROM against gravity

$4=$ active movement, full ROM against moderate resistance

$5=($ normal $)$ active movement, full ROM against full resistance

$\mathrm{NT}=$ not testable

The following muscles are to be examined (bilaterally) and graded using the scale defined above. These muscles were chosen because of their consistency for being innervated by the segments indicated and their ease of testing in the clinical situation, where testing in any position other than the supine position may be contraindicated.

C5-Elbow flexors (biceps, brachialis)

C6-Wrist extensors (extensor carpi radialis longus and brevis)

C7-Elbow extensors (triceps)

C8-Finger flexors (flexor digitorum profundus) to the middle finger

$\mathrm{T} 1-$ Small finger abductors (abductor digiti minimi)

L2 - Hip flexors (iliopsoas)

L3-Knee extensors (quadriceps)

L4-Ankle dorsiflexors (tibialis anterior)

L5-Long toe extensors (extensor hallucis longus)

S1-Ankle plantarflexors (gastrocnemius, soleus) 
In addition to bilateral testing of these muscles, the external anal spincter should be tested on the basis of contractions around the examiner's finger and graded as being present or absent (i.e., enter Yes or No on the patient's summary sheet). This latter information is used solely for determining the completeness of injury.

\section{Motor examination: optional elements}

For purposes of SCI evaluation, it is recommended that other muscles be evaluated, but their scores are not used in determining the motor score, motor level or completeness. As warranted, it is suggested that the following muscles be tested: (1) diaphragm, (2) deltoid and (3) lateral hamstrings. Their strength is to be rated as absent, weak or normal.

\section{Sensory and motor scores/levels}

Sensory scores and sensory level

Required testing generates four sensory modalities per dermatome: R-pin prick, R-light touch, L-pin prick, L-light touch. As is indicated on the summary chart these scores are then summed across dermatomes and sides of body to generate two summary sensory scores: pin prick and light touch score. The sensory scores provide a means of numerically documenting changes in sensory function.

Further, through the required sensory examination the sensory components for determining neurological level (i.e., the sensory level), zone of partial preservation and impairment grade are obtained.

\section{Motor scores and motor level}

The required motor testing generates two motor grades per paired myotome: right and left. As is indicated on the summary chart, these scores are then summed across myotomes and sides of body to generate a single summary motor score. The motor score provides a means of numerically documenting changes in motor function.

Further, through the required motor examination, the motor components for determining neurological level (i.e., the motor level), zone of partial preservation and impairment grade are obtained.

\section{Motor level determination: further considerations}

Just as each segmental nerve (root) innervates more than one muscle, most muscles are innervated by more than one nerve segment (usually two segments; see Figure $2)$. Therefore, the assigning of one muscle or one muscle group (i.e., the key muscle) to represent a single spinal nerve segment is a simplification, used with the understanding that in any muscle the presence of innervation by one segment and the absence of innervation by the other segment will result in a weakened muscle.

By convention, if a muscle has at least a grade of 3 , it is considered to have intact innervation by the more rostral of the innervating segments. In determining the motor level, the next most rostral key muscle must test as 4 or 5 , since it is assumed that the muscle will have both of its two innervating segments intact. For example, if no activity is found in the $\mathrm{C} 7 \mathrm{key}$ muscle and the $\mathrm{C} 6$ muscle is graded 3, then the motor level for the tested side of the body is $\mathrm{C} 6$, providing the $\mathrm{C} 5$ muscle is graded at least 4 .

The examiner's judgment is relied upon to determine whether a muscle that is graded at least 4 is fully innervated. This is necessary because a number of factors may, in some patients, inhibit a full effort during clinical testing at varying times post-injury. Examples include pain, position of the patient, hypertonicity and disuse. A grade 4 should not be considered normal if the examiner feels none of these inhibiting factors is present and the patient is exerting a full effort, yet only produces a grade 4 in that muscle.

In short, the motor level (the lowest normal motor segment-which may differ by side of body) is defined by the lowest key muscle that has a grade of at least 3, providing the key muscles represented by segments above that level are judged to be normal (4 or 5$)$. 


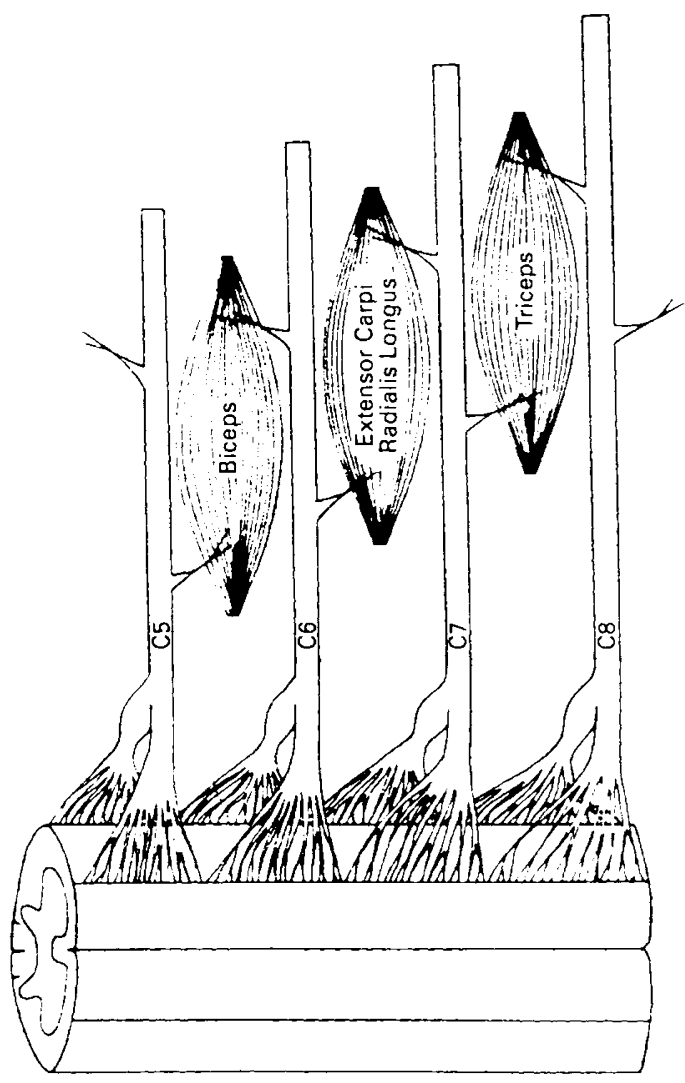

Figure 2 Schematic depiction of innervation of each of three key muscles by two nerve segments.

\section{ASIA Impairment Scale (modified from Frankel)}

The following scale is used in grading the degree of impairment:

$A=$ Complete. No sensory or motor function is preserved in the sacral segments S4-S5.

$B=$ Incomplete. Sensory but not motor function is preserved below the neurological level and extends through the sacral segments S4-S5.

$C=$ Incomplete. Motor function is preserved below the neurological level, and the majority of key muscles below the neurological level have a muscle grade less than 3 .

$D=$ Incomplete. Motor function is preserved below the neurological level, and the majority of key muscles below the neuro- logical level have a muscle grade greater than or equal to 3 .

$E=$ Normal. Sensory and motor function is normal.

\section{Clinical syndromes}

Central cord syndrome

A lesion, occurring almost exclusively in the cervical region, that produces sacral sensory sparing and greater weakness in the upper limbs than in the lower limbs.

\section{Brown-Séquard syndrome}

A lesion that produces relatively greater ipsilateral proprioceptive and motor loss and contralateral loss of sensitivity to pin and temperature.

\section{Anterior cord syndrome}

A lesion that produces variable loss of motor function and of sensitivity to pin and temperature, while preserving proprioception.

\section{Conus medullaris syndrome}

Injury of the sacral cord (conus) and lumbar nerve roots within the neural canal, which usually results in an areflexic bladder, bowel and lower limbs, with lesions as at B in Figure 3. Sacral segments may occasionally show preserved reflexes, e.g., bulbocavernosus and micturition reflexes, with lesions as at $\mathrm{A}$ in Figure 3.

\section{Cauda equina syndrome}

Injury to the lumbosacral nerve roots within the neural canal resulting in areflexic bladder, bowel and lower limbs, with lesions as at $\mathrm{C}$ in Figure 3.

\section{Functional Independence Measure (FIM)}

To fully describe the impact of SCI on the individual and to monitor/evaluate progress associated with treatment, a standard measure of daily-life activities is necessary. The Functional Independence Measure (FIM) is one approach to functional assessment that has become widely utilized in the 


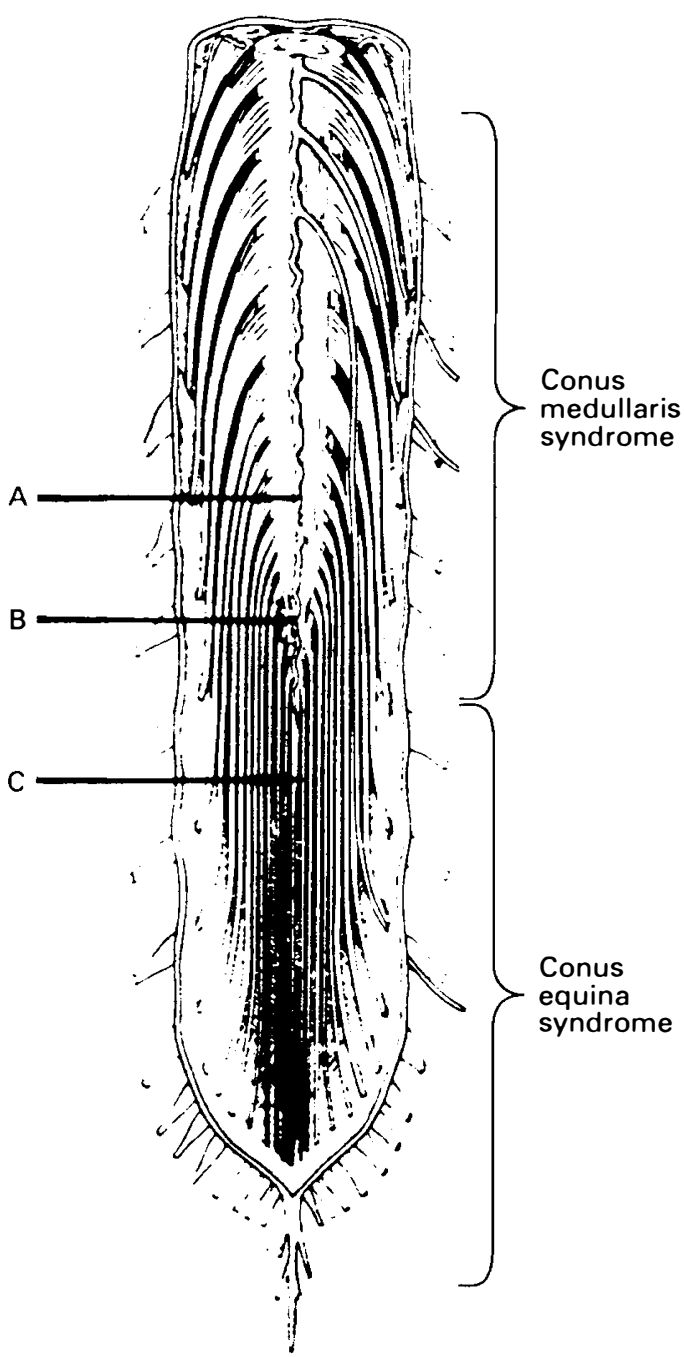

Figure 3 Conus medullaris and cauda equina syndromes.

US and is gaining acceptance internationally.

The FIM focuses on six areas of functioning: self care, sphincter control, mobility, locomotion, communication and social cognition. Within each area, two or more specific activities/items are evaluated, with a total of 18 items. For example, six activity items (eating, grooming, bathing, dressing upper body, dressing lower body, and toileting) comprise the self-care area (see summary chart).

Each of the 18 items is evaluated in terms of independence of functioning, using a seven-point scale.

Independent (no human assistance is required)

7 = Complete independence: the activity is typically performed safely, without modification, assistive devices or aids, and within reasonable time.

6 = Modified independence: the activity requires an assistive device and/or more than reasonable time and/or is not performed safely.

Dependent (human supervision or physical assistance is required)

5 = Supervision or setup: no physical assistance is needed, but cuing, coaxing or setup is required.

$4=$ Minimal contact assistance: subject requires no more than touching and expends $75 \%$ or more of the effort required in the activity.

$3=$ Moderate assistance: subject requires more than touching and expends $50-75 \%$ of the effort required in the activity.

$2=$ Maximal assistance: subject expends $25-50 \%$ of the effort required in the activity.

$1=$ Total assistance: subjects expends $0-25 \%$ of the effort required in the activity.

Thus, the FIM total score (summed across all items) estimates the cost of disability in terms of safety issues and of dependence on others and on technological devices. The profile of area scores and item scores pinpoints the specific aspects of daily living that have been most affected by SCI.

In using the FIM with individuals who have experienced SCI, it should be kept in mind that the FIM was developed for the disabled population in general. It samples those areas of activity that have been found to be affected by impairment among diverse disability groups. Although basic issues of reliability and validity of the FIM have been explored by the developers, its validity as an instrument for precisely gauging changed functioning with all SCI subpopulations has yet to be demonstrated empirically. For 
example, it is not yet clear that the self-care items sensitively gauge changes in self-care functioning experienced by tetraplegics during the course of rehabilitation. Further, the reliability estimates for the communication and social cognition areas have been found to be lower than for other areas assessed. Despite these caveats, the use of the FIM is recommended, as it is relatively simple to use, reflects functional issues of importance to SCI, and guidelines for its use have been carefully developed.

Specific instructions for use of the FIM can be obtained directly from the developers of the FIM. Ask for the Guide for Use of the Uniform Data Set for Medical Rehabilitation (1990), at the following address: Center for Functional Assessment Research, State University of New York, Buffalo, NY 14260 USA.

\section{Bibliography}

1 Michaelis LS (1969) International inquiry on neurological terminology and prognosis in paraplegia and tetraplegia. Paraplegia 7: 1-5.

2 Ditunno JF (1992) Functional assessment measures in CNS trauma. J Neurotrauma 9: S301-S305.

3 Ditunno JF, Stover SL, Freed MM, Ahn JH (1992) Motor recovery of the upper extremities in traumatic quadriplegia: a multi-center study. Arch Phys Med Rehabil 73: 431-436.

4 Bracken MB, Shepard MJ, Collins WF et al (1990) A randomized, controlled trial of methylprednisolone or naloxone in the treatment of acute spinal cord injury. $N$ Engl J Med 322: 1405-1411.

5 Bracken MB, Shepard MJ, Collins WF et al (1992) Methylprednisolone or naloxone treatment after acute spinal cord injury: 1 year follow-up data. J Neurosurg 76: 23-31.

6 Hamilton BB, DeVivo MJ (1990) Functional enhancement. In: Spinal Cord Injury: The Model. Proceedings of a National Consensus Conference on Catastrophic Illness and Injury. Georgia Regional Spinal Cord Injury Care System. Shepherd Center for Treatment of Spinal Injuries, Atlanta, GA.

7 American Spinal Cord Injury Association (1982) Standards for Neurological Classification of Spinal Injured Patients. ASIA, Chicago.

8 Long CC, Lawton EB (1955) Functional significance of spinal cord lesion level. Arch Phys Med Rehabil 46: 249-255.

9 Stover SL, Fine PR (1986) Spinal Cord Injury: The Facts and Figures. The University of Alabama at Birmingham, Birmingham, AL.

10 Ditunno JF (1992) New spinal cord injury standards - 1992. Paraplegia 30: 90-91.

11 Walker MD (1991) Acute spinal cord injury. $N$ Engl J Med 324: 1885-1888.

12 ASIA/IMSOP (1992) International Standards for Neurological and Functional Classification of Spinal Cord Injury - Revised 1992. American Spinal Injury Association, Chicago.

\section{Appendix I}

Affiliations of committee members participating in developing the standards recommended herein:

American Academy of Orthopaedic Surgery (AAOS)

American Academy of Physical Medicine and

Rehabilitation (AAPM\&R)

American Association of Neurological Surgery (AANS)

American Association for Surgery of Trauma
(AAST)

American College of Epidemiology

American Congress of Rehabilitation Medicine (ACRM)

American Congress of Surgery (ACS)

American Spinal Injury Association (ASIA)

Congress of Neurological Surgery (CNS)

International Medical Society of Paraplegia (IMSOP)

Joint Section on Neurotrauma and Critical Care of AANS/CNS

The Neurotrauma Society 
STANDARD NEUROLOGICAL CLASSIFICATION OF SPINAL CORD INJURY MOTOR
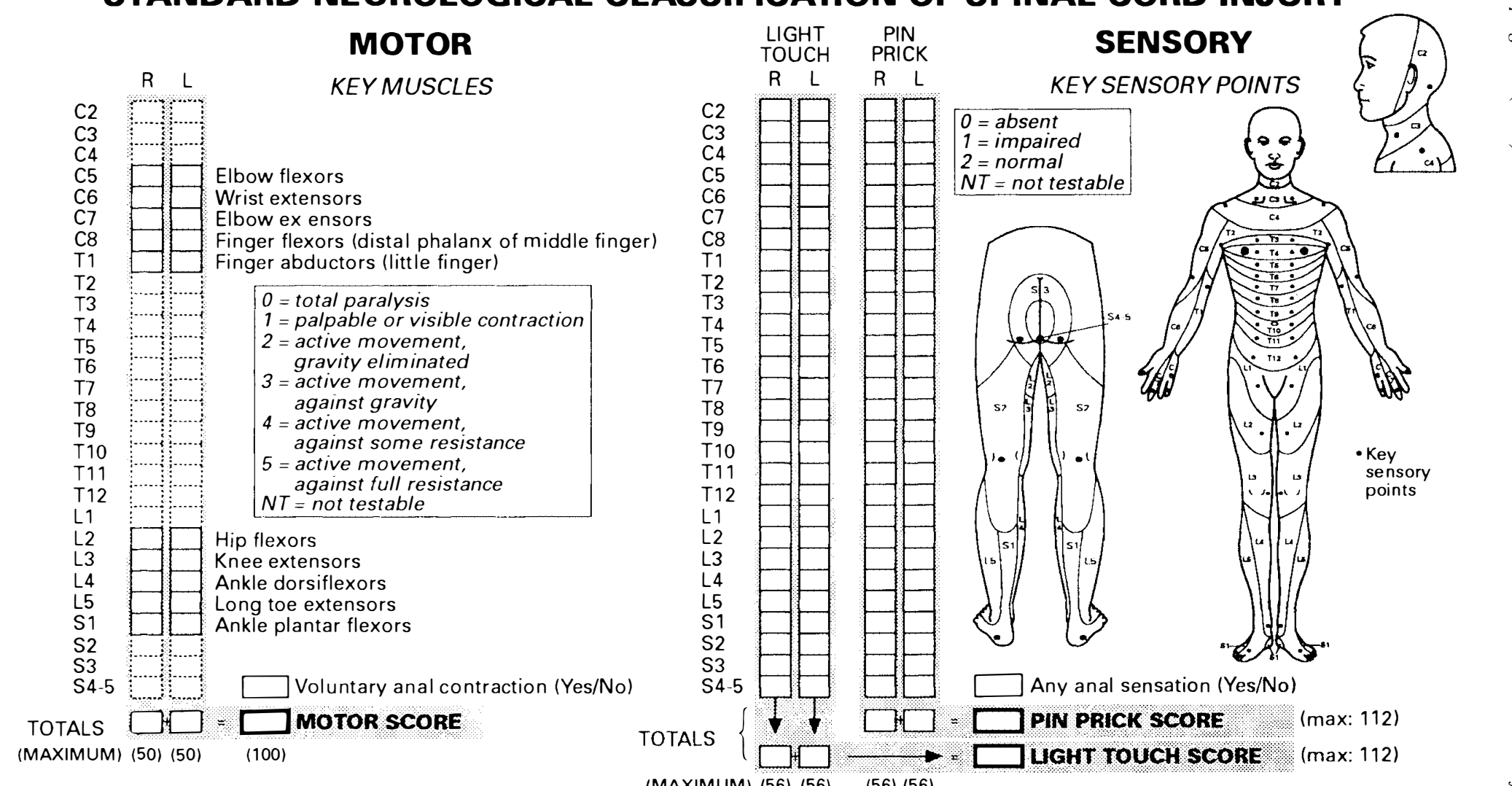

\section{NEUROLOGICAL LEVEL}

The most caudat segment

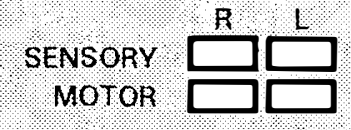

\section{COMPLETE OR \\ INCOMPLETE?}

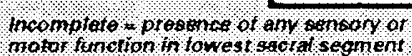

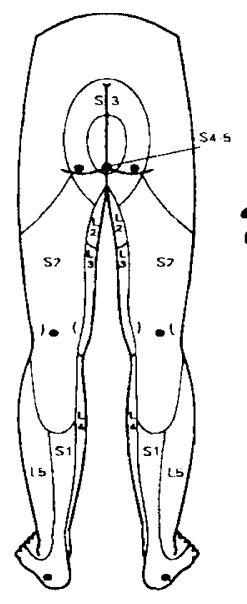

Any anal sensation (Yes/No)

PIN PRICK SCORE

(max: 112)

JuGHT rOUCH SCORI? : (max: 112)

(56) $(56)$

This form may be copied freely but not altered without permission from the American Spinal Injury Association

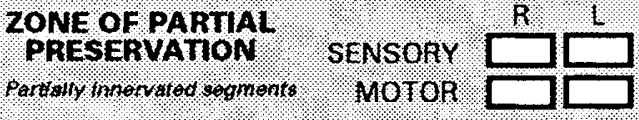
Version 4 d 


\section{Functional Independence Measure (FIM)}

\begin{tabular}{|c|c|c|}
\hline & $\begin{array}{l}7 \text { Complete Independence (Timely, Safely) } \\
6 \text { Modified Independence (Device) }\end{array}$ & $\begin{array}{l}\text { No } \\
\text { Helper }\end{array}$ \\
\hline $\begin{array}{l}\mathrm{L} \\
\mathrm{E} \\
\mathrm{V} \\
\mathrm{E} \\
\mathrm{L}\end{array}$ & $\begin{array}{l}\text { Modified Dependence } \\
5 \text { Supervision } \\
4 \text { Minimal Assist }(\text { Subject }=75 \%+) \\
3 \text { Moderate Assist }(\text { Subject }=50 \%+) \\
\text { Complete Dependence } \\
2 \text { Maximal Assist }(\text { Subject }=25 \%+) \\
1 \text { Total Assist }(\text { Subject }=0 \%+)\end{array}$ & Helper \\
\hline
\end{tabular}

\section{Self Care}

A. Eating

B. Grooming

C. Bathing

D. Dressing-Upper Body

E. Dressing-Lower Body

F. Toileting

\section{Sphincter Control}

G. Bladder Management

H. Bowel Management

Mobility

Transfer:

I. Bed, Chair, Wheelchair

J. Toilet

K. Tub, Shower

Locomotion

L. Walk/wheelchair

M. Stairs

Communication

N. Comprehension

O. Expression

Social Cognition

P. Social Interaction

Q. Problem Solving

R. Memory

Total FIM

NOTE: Leave no blanks; enter 1 if patient not testable due to risk.

\section{COPY FREELY - DO NOT CHANGE}

RESEARCH FOUNDATION OF THE STATE UNIVERSITY OF NEW YORK

\section{ASIA Impairment Scale}

$A=$ Complete: No motor or sensory function is preserved in the sacral segments S4-S5.

$B=$ Incomplete: Sensory but not motor function is preserved below the neurological level and extends through the sacral segments S4-S5.

$C=$ Incomplete: Motor function is preserved below the neurological level, and the majority of key muscles below the neurological level have a muscle grade less than 3.

$D=$ Incomplete: Motor function is preserved below the neurological level, and the majority of key muscles below the neurological level have a muscle grade greater than or equal to 3 .

$E=$ Normal: Motor and sensory function is normal.

\section{Clinical syndromes}

Central cord

Brown-Séquard

Anterior cord

Conus medullaris

Cauda equina 